\title{
Spin-Resolved Spectroscopy of the Yu-Shiba-Rusinov States of Individual Atoms
}

\author{
L. Cornils, ${ }^{1}$ A. Kamlapure, ${ }^{1, \dagger}$ L. Zhou, ${ }^{1,}$ S. Pradhan, ${ }^{2}$ A. A. Khajetoorians, ${ }^{1,}$ \\ J. Fransson, ${ }^{2}$ J. Wiebe, ${ }^{1, *}$ and R. Wiesendanger ${ }^{1}$ \\ ${ }^{1}$ Department of Physics, Hamburg University, D-20355 Hamburg, Germany \\ ${ }^{2}$ Department of Physics and Astronomy, Uppsala University, P.O. Box 516, Uppsala SE-751 21, Sweden
}

(Received 21 June 2017; published 9 November 2017)

\begin{abstract}
A magnetic atom in a superconducting host induces so-called Yu-Shiba-Rusinov (YSR) bound states inside the superconducting energy gap. By combining spin-resolved scanning tunneling spectroscopy with simulations we demonstrate that the pair of peaks associated with the YSR states of an individual Fe atom coupled to an oxygen-reconstructed Ta surface gets spin polarized in an external magnetic field. As theoretically predicted, the electron and hole parts of the YSR states have opposite signs of spin polarizations which keep their spin character when crossing the Fermi level through the quantum phase transition. The simulation of a YSR state right at the Fermi level reveals zero spin polarization which can be used to distinguish such states from Majorana zero modes in chains of YSR atoms.
\end{abstract}

DOI: 10.1103/PhysRevLett.119.197002

Recent investigations of chains [1-3] and arrays [4,5] of magnetic atoms in contact with surfaces of $s$-wave superconductors in view of Majorana zero modes and topological superconductivity triggered renewed interest in the properties of the basic constituent of such systems, the individual magnetic adatom. Typically, such adatoms induce quasiparticle excitations inside the superconducting energy gap [6-8], referred to as Yu-Shiba-Rusinov (YSR) states, which hamper the identification of topologically nontrivial edge states [3,9], calling for a thorough characterization of all experimentally accessible properties of YSR states.

Following the first experimental verification of YSR states of individual atoms using scanning tunneling spectroscopy (STS) [10], there were numerous experimental studies, focusing on orbital effects [11-13], magnetic anisotropy [14], effects of reduced dimensionality of the superconductor [15], effects of coupling [11,16], and the competition between Kondo screening and superconducting pairing $[17,18]$. However, the investigation of the spin polarization of the YSR state of individual atoms, which could serve as a fingerprint for the distinction from topological states [19], was so far restricted to theoretical predictions [6-8,20-22].

Neglecting orbital effects or magnetic anisotropy, theory predicts one pair of spin-polarized states of a YSR atom, an electron $\left(e^{-}\right)$- and a hole $(h)$-like part with opposite spin character, which are located at $\pm|\varepsilon|$ from the Fermi level $E_{F}$ [20]. There is a pronounced asymmetry in spectral weight between the $e^{-}$and $h$ parts due to electron-hole asymmetry of the band structure $[21,22]$ and/or a nonmagnetic scattering potential of the impurity [20,23]. With increasing exchange coupling $J$ between the adatom and substrate, the binding energy $\varepsilon$ decreases until the YSR states eventually cross $E_{F}$ through a quantum phase transition (QPT), accompanied by an inversion of the asymmetry in the $e^{-}-h$ spectral weight [17]. Most notably, since both parts of the YSR states keep their spin character, the spin polarization above and below $E_{F}$ is expected to invert [20] through the QPT. Here, we experimentally verify these predictions for single $\mathrm{Fe}$ atoms on the surface of $\mathrm{Ta}(100)-(3 \times 3) \mathrm{O}$ combined with simulations. Our results prove that the $e^{-}-h$ inversion of the spin polarization is a characteristic fingerprint of YSR states, which enables us to differentiate between a YSR state right at $E_{F}$ and a Majorana zero mode of a chain of YSR atoms.

All experiments were performed under ultrahigh vacuum conditions in a SPECS scanning tunneling microscope (STM) facility [24] at $T=1.1 \mathrm{~K}$, where the magnetic field $B_{\text {ext }}$ is applied perpendicular to the sample surface. Details of sample and tip preparation, and of the YSR simulation method [25,26] are described in Ref. [27]. We used junction resistances of $V_{\text {stab }} / I_{\text {stab }} \approx 20 \mathrm{M} \Omega$, where effects due to Josephson tunneling and Andreev reflections do not play any role $[32,33]$.

The substrate preparation results in the formation of a $\mathrm{Ta}(100)-(3 \times 3) \mathrm{O}$ reconstruction [34] [Fig. 1(a) and [27]], which appears as a regular network of depression lines along [010] and [001] with an apparent depth of 50 pm surrounding circular and cross-shaped plaquettes. Both types of plaquettes have a multitude of faintly different appearances, probably indicating the presence of different metastable configurations of the reconstruction. STS reveals a superconducting energy gap $\Delta_{\text {sample }} \approx 0.63 \mathrm{meV}$ of the substrate [Fig. 1(e)] with a critical temperature $T_{\mathrm{c}} \approx 4 \mathrm{~K}$ [27] slightly smaller than the previously determined values $(\Delta=$ $0.71 \pm 0.02 \mathrm{meV}, T_{\mathrm{c}}=4.5 \pm 0.03 \mathrm{~K}$ ) from planar tunnel junctions and resistivity measurements $[35,36]$.

Cold deposition of Fe results in a statistical distribution of single Fe adatoms, mostly adsorbed on top of the center of the plaquettes [Fig. 1(b)], which we will focus on in the following. Because of the different configurations of the $\mathrm{Ta}(100)-(3 \times 3) \mathrm{O}$ reconstruction, there is a variety of slightly different adsorption geometries, which can be 

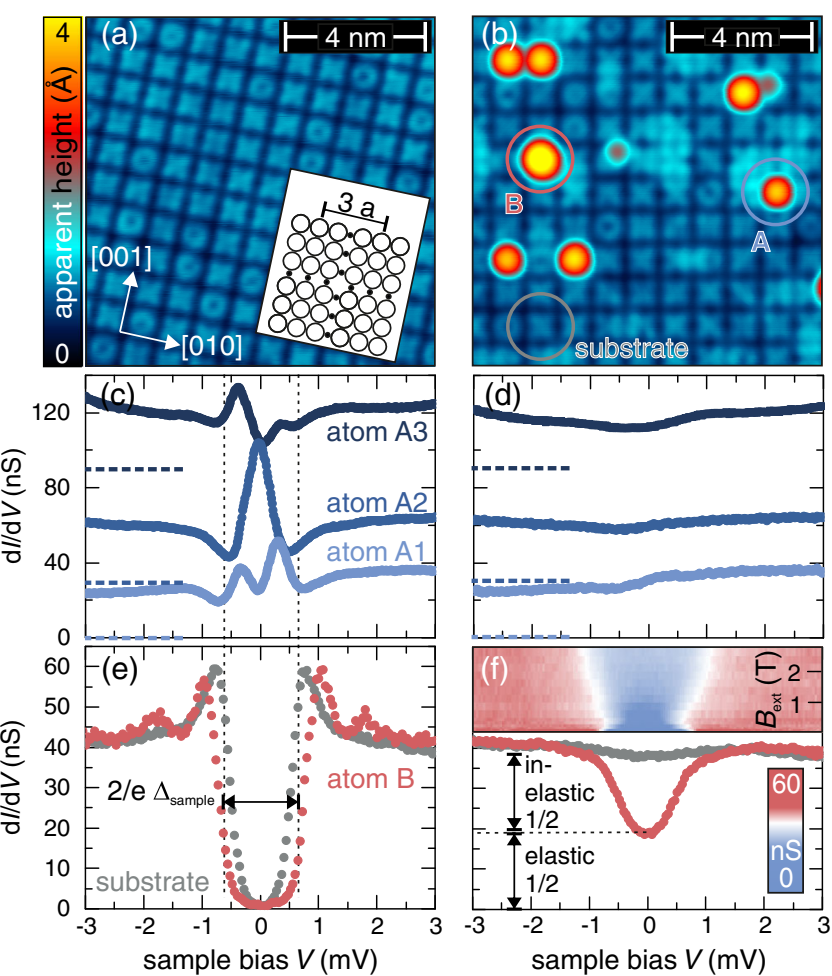

FIG. 1. (a),(b) Constant-current images of the $\mathrm{Ta}(100)$ $(3 \times 3) \mathrm{O}$ surface (a) before $(V=100 \mathrm{mV}, I=100 \mathrm{pA})$ and (b) after deposition of $\mathrm{Fe}(V=50 \mathrm{mV}, I=100 \mathrm{pA})$. The inset of (a) shows a model of the surface [34] with Ta and $\mathrm{O}$ atoms indicated by open and closed circles, respectively ( $a=0.33 \mathrm{~nm}$ ). Two types of adatoms $(A, B)$ and a substrate spot are indicated in (b). (c), (d) STS curves taken on three type $A$ Fe atoms at $B_{\text {ext }}=0$ (c) and $B_{\text {ext }}=0.5 \mathrm{~T}$ (d). The spectra are vertically offset for clarity as indicated by horizontal dashed zero lines $\left(V_{\text {stab }}=\right.$ $6 \mathrm{mV}, I_{\text {stab }}=300 \mathrm{pA}, V_{\text {mod }}=100 \mu \mathrm{V}$ ). (e),(f) STS curves taken on the substrate and on a type $B \mathrm{Fe}$ atom at $B_{\text {ext }}=0$ (e) and $B_{\text {ext }}=0.5 \mathrm{~T}$ (f). The inset in (f) shows a color plot of the $B_{\text {ext }}$-dependent STS of this atom $\left(V_{\text {stab }}=4 \mathrm{mV}, I_{\text {stab }}=200 \mathrm{pA}\right.$, $\left.V_{\text {mod }}=50 \mu \mathrm{V}\right)$. The dashed vertical lines in (c),(e) indicate the $\Delta_{\text {sample }}$.

identified by the adatoms' apparent heights [27] and their spectroscopic signatures [Figs. 1(c)-(f)]. The majority of shallower adatoms $(\sim 240 \mathrm{pm})$ have spectra that are indistinguishable from the substrate [27]. However, 9\% of those adatoms [e.g., type $A$ adatom in Fig. 1(b)] reveal peaks inside the superconducting gap accompanied by a suppression of the coherence peaks [Fig. 1(c)]. Different type $A$ atoms with different local substrate environments either [atom $A 2$ in Fig. 1(c)] have one broader peak at the bias approximately corresponding to $E_{F}(V=0 \mathrm{~V})$, or a pair of peaks (atom $A 1$ ) at voltages $\pm \frac{\varepsilon}{e}$ symmetric around $E_{F}$ with larger intensity of the unoccupied state $\left(\mathcal{I}_{\mathrm{e}}\right.$ at $V>0)$ than that of the occupied state $\left(\mathcal{I}_{\mathrm{h}}\right.$ at $\left.V<0\right)$, or a similar pair of peaks but with reversed order of intensities (atom A3). When the superconductivity of the substrate is quenched using $B_{\text {ext }}=0.5 \mathrm{~T}$ [gray curve in Fig. 1(f)], these in-gap states disappear [Fig. 1(d)]. This behavior is indicative of YSR states with different $\varepsilon$ [17], and more evidence for this conclusion is provided below.

Spectra taken on the higher adatoms [ 390 pm, e.g., type $B$ adatom in Fig. 1(b)] reveal replicas of the coherence peaks at higher bias voltage [Fig. 1(e)]. When superconductivity is quenched, the spectra show steps at $\pm 0.5 \mathrm{mV}$ symmetrically around $E_{F}$, which are monotonically shifting to higher bias for increasing $B_{\text {ext }}$ [Fig. 1(f)]. These are the fingerprints of a spin excitation of a magnetic atom on a superconducting substrate $[25,26,37,38]$. Note that the absence of bound states inside the superconducting gap and the large fraction of inelastic tunneling events of about 50\% indicate a negligible exchange coupling of these adatoms with the superconductor. Indeed, for quenched superconductivity, the $B_{\text {ext }}$-dependent spectra can be excellently simulated using the effective spin model from Ref. [39] with the effective spin Hamiltonian $\hat{H}=g \mu_{B} \hat{S}_{z} B_{\text {ext }}+D \hat{S}_{z}^{2}+E\left(\hat{S}_{x}^{2}-\hat{S}_{y}^{2}\right)$ without a significant Kondo scattering parameter. Here, $\hat{S}_{z}$ is the operator of the out-of-plane component of the adatom's effective spin, $g$ is the Lande $g$ factor, $\mu_{B}$ is the Bohr magneton, and $D$ and $E$, respectively, are the axial and transverse magnetic anisotropy constants. The best fits are achieved for the parameters $S=2, g=2, \quad D=$ $-0.247 \mathrm{meV}$, and $E=0.133 \mathrm{meV}$ [27]. We will later use this type of adatom to calibrate the tip's spin polarization for spin-resolved scanning tunneling spectroscopy (SPSTS) measurements on YSR (type $A$ ) adatoms.

We now turn to a spectroscopic investigation of the YSR adatoms with higher energy resolution using a superconducting tip. STS curves of the substrate and about ten type $A$ atoms with different local environments of the reconstruction are shown in Fig. 2(a) and Ref. [27]. Because of the additional gap of the tip, the coherence peaks of the substrate spectrum now appear at $\pm 1 / e\left(\Delta_{\text {sample }}+\Delta_{\text {tip }}\right)$, which is used to determine the tip's energy gap $\Delta_{\text {tip }} \approx 0.37 \mathrm{meV}$ from the known $\Delta_{\text {sample. }}$. On the type $A$ atoms, the pair of most intense in-gap peaks appears at $\pm 1 / e\left(\Delta_{\text {tip }}+|\varepsilon|\right)$, with an asymmetry $A_{e-h}=$ $\mathcal{I}_{e}-\mathcal{I}_{h}$ in the peak intensities of the $e^{-}$- and $h$-like states similar to the observation of Fig. 1(c). Note that the additional weaker pair of peaks at $\pm 1 / e\left|\Delta_{\text {tip }}-\right| \varepsilon||$ is due to tunneling into the same bound state by thermally occupied tip states $[17,33]$. Within the energy resolution of this experiment, we do not see indications for additional states. The type $A$ atoms can be divided into three categories [Fig. 2(a)], i.e., $A_{e-h}>0, \varepsilon>0$ (atom 1, we define the sign of $\varepsilon$ from the energetic position of the more intense bound state), $\varepsilon \approx 0$ (atom 2), and $A_{e-h}<0, \varepsilon<0$ (atom 3). The extracted asymmetries for all investigated atoms are plotted in Fig. 2(b) against $\varepsilon$. This behavior is consistent with that of YSR bound states of atoms with varying $J$ across the QPT as observed for magnetic molecules adsorbed on a superconductor [17]. Indeed, the asymmetries and peak positions are quantitatively 
$-\left(\Delta_{\text {tip }}+|\varepsilon|\right) / e \quad\left(\Delta_{\text {tip }}+|\varepsilon|\right) / e$
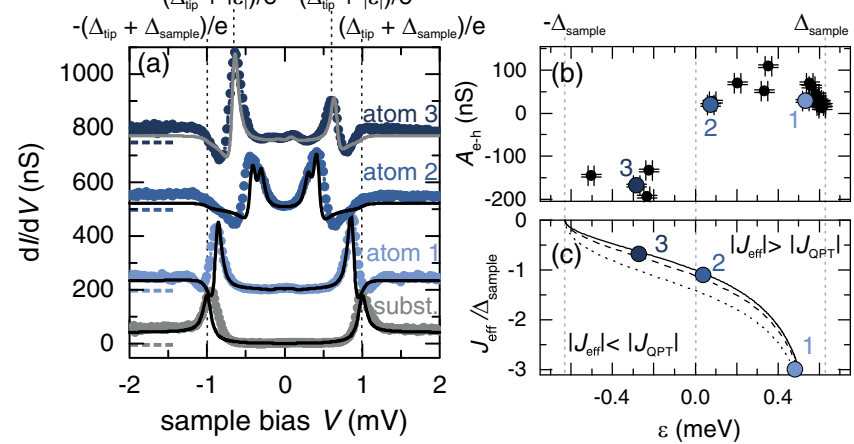

FIG. 2. (a) Points show STS curves taken with a superconducting tip at $B_{\text {ext }}=0 \mathrm{~T}$ on the substrate and on three YSR Fe atoms (type $A$ ). Lines show simulated spectra assuming different $J_{\text {eff }}$ given in (c) and $U=-0.1$ (atoms 1,2), and $U=-0.4$ (atom 3). The spectra are vertically offset for clarity as indicated by horizontal dashed zero lines. The dashed vertical lines indicate $\left(\Delta_{\text {sample }}+\Delta_{\text {tip }}\right)$ determined from the substrate spectrum, and $\varepsilon$ exemplarily for atom $3\left(V_{\text {stab }}=10 \mathrm{mV}, \quad I_{\text {stab }}=600 \mathrm{pA}\right.$, $V_{\text {mod }}=20 \mu \mathrm{V}$ ). (b) $A_{e-h}$ versus $\varepsilon$ extracted from the experimental data in (a) (points with according numbers) and additional data [27]. (c) Points show data pairs $J_{\text {eff }}, \varepsilon$ used for the simulations in (a). The lines give the calculated $J_{\text {eff }}(\varepsilon)$ for $U=0$ (solid), $U=-0.5$ (dashed), and $U=-1$ (dotted).

reproduced by YSR simulations which consider the effective exchange interaction $J_{\text {eff }}$ between adatom and substrate and a scattering potential strength $U$ [27] assuming a variation of $J_{\text {eff }}$, and keeping the same sign of $U$ [Fig. 2(a)]. The according parameters of $J_{\text {eff }}$ versus $\varepsilon$ are given in Fig. 2(c) together with the simulated $J_{\text {eff }}(\varepsilon)$ for different $U$ illustrating the QPT at $J_{\mathrm{QPT}} / \Delta_{\text {sample }}=-\sqrt{1+U^{2}}$ between the two different regimes $\left|J_{\text {eff }}\right|<\left|J_{\mathrm{QPT}}\right|$ and $\left|J_{\text {eff }}\right|>\left|J_{\mathrm{QPT}}\right|$, which is driven by a variation of the local substrate environment of the type $A$ atoms.

To study the spin polarization of the YSR states on different sides of this QPT, we investigated type $A$ atoms with a magnetic tip (see below) which was beforehand characterized by SPSTS on a type $B$ atom (Fig. 3). SPSTS spectra were acquired as a function of $B_{\text {ext }}$ which was swept down from +1.5 to $-1.5 \mathrm{~T}$, and then back up to $+1.1 \mathrm{~T}$ [Fig. 3(a) and Ref. [27] for the full data set]. The SPSTS spectra reveal a considerable asymmetry in the intensities at positive and negative bias above the excitation threshold, which is not observed using a nonpolarized tip [Fig. 1(f)], and reflects the spin-dependent tunneling between the spinpolarized tip and the magnetized atom [40]. The asymmetry inverts between -0.9 and $-1.0 \mathrm{~T}$ in the down sweep (curves 1 and 2), and again between +0.9 and $+1.0 \mathrm{~T}$ in the up sweep (curves 3 and 4), accompanied by a significant decrease in the excitation energy. The full behavior of the asymmetry is revealed by plotting $d I / d V(V=-4 \mathrm{mV})$ extracted for all spectra versus $B_{\text {ext }}$ [Fig. 3(b)]. In the normal conducting state of the substrate it resembles an $s$-shaped magnetization curve [41], which is
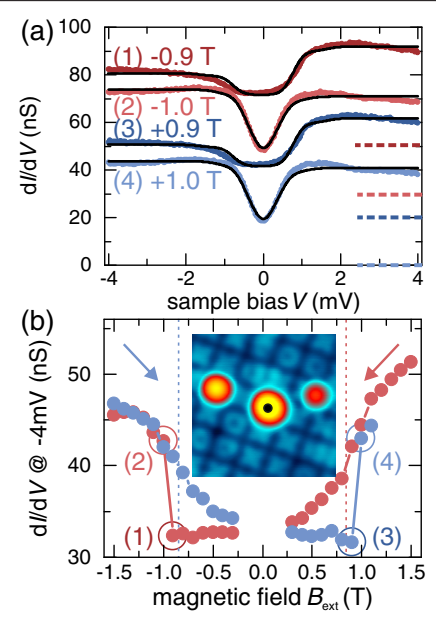

(c) (1) down sweep (2) down sweep

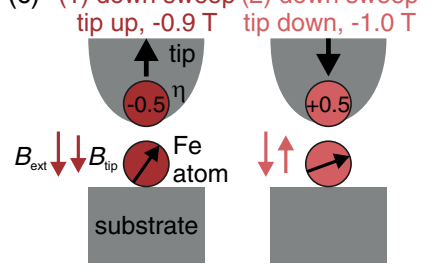

(3) up sweep (4) up sweep tip down, +0.9 T tip up, +1.0T

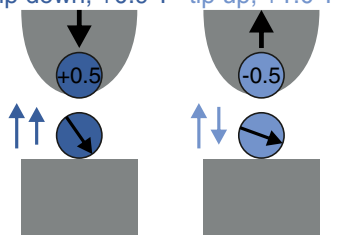

FIG. 3. (a) Colored lines are SPSTS curves of a type $B$ Fe atom [see the atom marked by a dot in the inset constant-current image of (b)] taken at the indicated $B_{\text {ext }}$ values when sweeping $B_{\text {ext }}$ downwards $(1,2)$ and back upwards $(3,4) \quad\left(V_{\text {stab }}=6 \mathrm{mV}\right.$, $\left.I_{\text {stab }}=300 \mathrm{pA}, V_{\text {mod }}=100 \mu \mathrm{V}\right)$. The spectra are vertically offset for clarity as indicated by horizontal dashed zero lines. The black lines are simulations within an effective spin model [39] using the parameters given in the text. (b) Magnetization curve extracted from the $d I / d V$ values at $-4 \mathrm{mV}$ of the SPSTS curves in (a) and Ref. [27]. The sweep direction is indicated by arrows. The circles indicate the points extracted from the SPSTS curves in (a). Dashed vertical lines indicate $B_{\text {tip }}= \pm 0.85$ T. (c) Sketch of the orientations of the background tip magnetization, $B_{\text {tip }}, B_{\text {ext }}$, the Fe spin $S$ (tilt schematically illustrates the changing saturation of the spin in the magnetic field direction), and the sign of $\eta$ for the four different cases $(1,2,3,4)$ following from the fits in (a).

shifted by $+0.85 \mathrm{~T}(-0.85 \mathrm{~T})$ for the down (up) sweep, and shows the same switching of the asymmetry between -0.9 and $-1.0 \mathrm{~T}(+0.9$ and $+1.0 \mathrm{~T})$ as observed in Fig. 3(a). We interpret the concurrent asymmetry switching and shift of excitation energy by a reversal of both the tip's spin polarization at $E_{F}, \eta=\left(n_{\uparrow}-n_{\downarrow}\right) /\left(n_{\uparrow}+n_{\downarrow}\right) \quad\left(n_{\uparrow, \downarrow}\right.$ are densities of spin-up and spin-down electrons), and the effective tip field $B_{\text {tip }}$ acting on the atom together with $B_{\text {ext }}$ [Fig. 3(c)]. Indeed, all magnetic field dependent SPSTS spectra can be quantitatively simulated [black lines in Fig. 3(a) and Ref. [27]] by assuming the same parameters of the adatom's effective spin Hamiltonian used for the nonpolarized measurements [Fig. 1(f)], but with $\eta= \pm 0.5$ and $B_{\text {tip }}= \pm 0.85 \mathrm{~T}$, which are simultaneously reversing between -0.9 and $-1.0 \mathrm{~T}(+0.9$ and $+1.0 \mathrm{~T})$ in the down (up) sweep. The according orientations of $B_{\text {ext }}, B_{\text {tip }}$, and of the Fe atom spin, and signs of $\eta$ are given in Fig. 3(c). Note, that $B_{\text {tip }}$ acts locally on the adatom without quenching the superconductivity in the substrate as shown by the preserved coherence peaks in the spectra for $B_{\text {ext }}=0 \mathrm{~T}$ [27]. Effective tip fields of a few tenths of a Tesla have been observed before in SPSTS of single atoms [41], and are most likely a result of an exchange interaction between the tip and the adatom. 

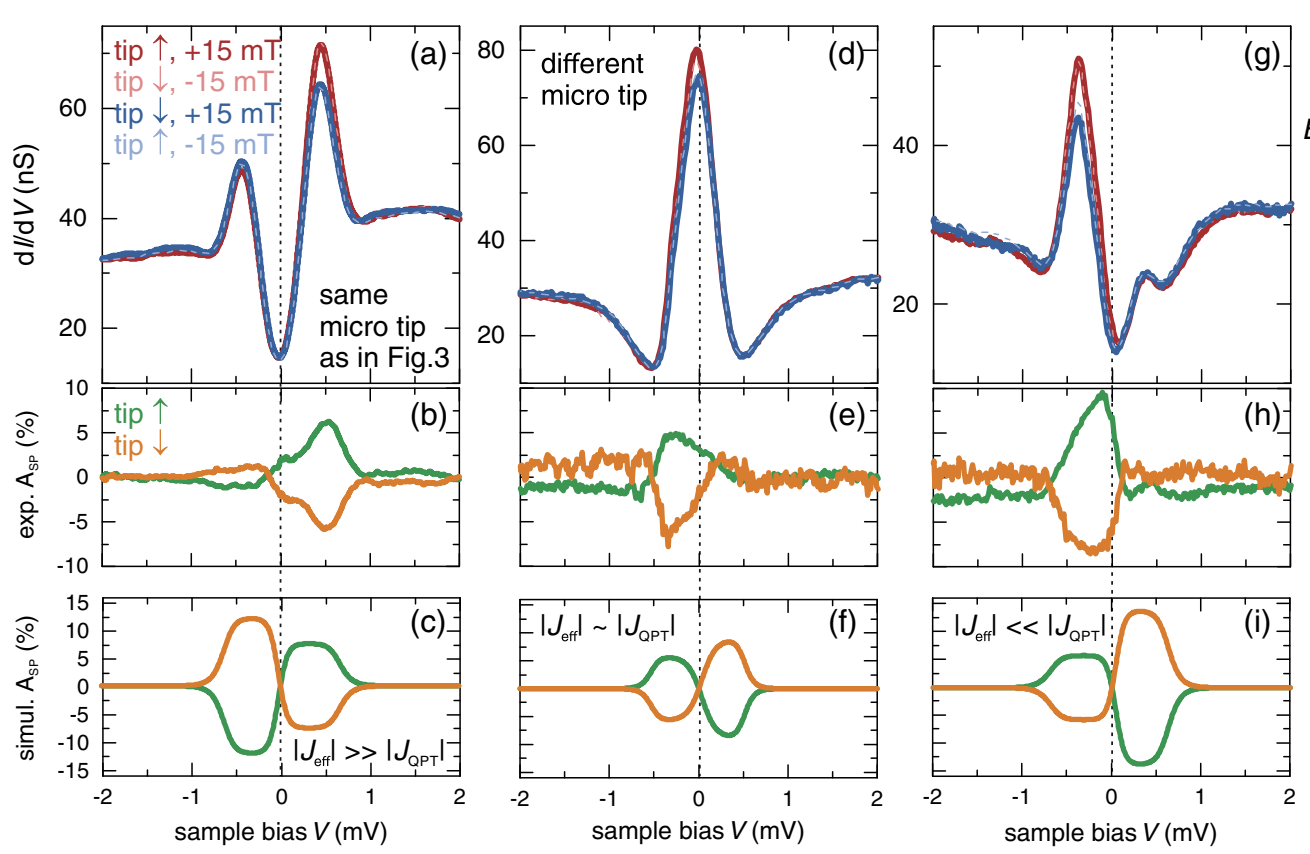

(j)
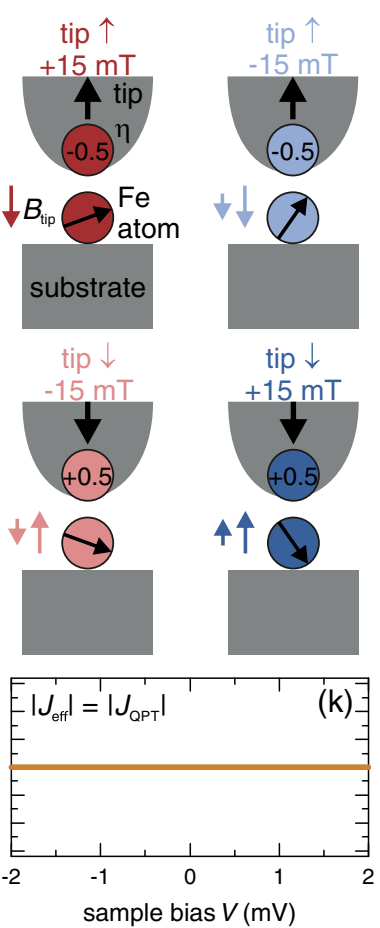

FIG. 4. (a) SPSTS using the same microtip characterized in Fig. 3 of the YSR state of a type $A$ Fe atom with $A_{e-h}>0$ at the given $B_{\text {ext }}$ and with orientations of the tip's magnetization indicated by arrows [see (j), $V_{\text {stab }}=6 \mathrm{mV}, I_{\text {stab }}=300 \mathrm{pA}, V_{\text {mod }}=100 \mu \mathrm{V}$ ]. (b) $A_{\mathrm{SP}}$ for upwards and downwards pointing tip magnetization extracted from (a). (c) Simulation of $A_{\mathrm{SP}}$ for the case of $-J_{\mathrm{eff}}=2 \Delta_{\text {sample }} \gg-J_{\mathrm{QPT}}$ $(U=-0.3)$. (d)-(i) Same as (a)-(c), but using a different microtip, and for an atom with a YSR state close to $E_{F}(\mathrm{~d}),(\mathrm{e})$, and $-J_{\text {eff }}=0.9 \Delta_{\text {sample }} \sim-J_{\mathrm{QPT}}(U=-0.3)(\mathrm{f})$, and for an atom with $A_{e-h}<0(\mathrm{~g}),(\mathrm{h})$, and $-J_{\text {eff }}=0.6 \Delta_{\text {sample }} \ll-J_{\mathrm{QPT}}(U=-0.6)(\mathrm{i})$. (j) Sketch of the orientations of the background tip magnetization, $\eta, B_{\text {tip }}, B_{\text {ext }}$, and of the Fe spin $S$ for the four different cases of (a). (k) Simulation of $A_{\mathrm{SP}}$ at the QPT.

Using the same microtip characterized in Fig. 3, SPSTS spectra were acquired on the YSR state of a type $A$ atom with $A_{e-h}>0$, i.e. $\left|J_{\text {eff }}\right| \gg\left|J_{\mathrm{QPT}}\right|$ [Fig. 4(a)]. We first apply $B_{\text {ext }}= \pm 1 \mathrm{~T}$ to orient the tip up or down $(\eta=\mp 0.5)$, respectively. Note that $B_{\text {tip }}$ and $\eta$ are oriented antiparallel to the tip [see Fig. 4(j)]. For SPSTS in both of these tip states, we apply $B_{\text {ext }}= \pm 15 \mathrm{mT}$ which is weak enough to keep the tip unaffected and the substrate superconducting, but should have a small effect on the adatom's spin. Indeed, the spectra in Fig. 4(a) reveal a clear difference in peak intensity of the $e^{-}$part $(V>0)$ of the YSR state when either $\eta$ or $B_{\text {ext }}$ are reversed, but no change when reversing both. This indicates a significant spin-polarization of the YSR state of the adatom stabilized by $B_{\text {tip }}+B_{\text {ext }}$. The extracted spin-asymmetries $A_{\mathrm{SP}}=\left(d I / d V_{+15 \mathrm{mT}}-\right.$ $\left.d I / d V_{-15 \mathrm{mT}}\right) /\left(d I / d V_{+15 \mathrm{mT}}+d I / d V_{-15 \mathrm{mT}}\right)$ for the two tip orientations [Fig. 4(b)] are $A_{\mathrm{SP}} \sim 5 \%$. For the $h$ part of the YSR state $(V<0), A_{\mathrm{SP}}$ is considerably weaker and reversed with respect to the $e^{-}$part. The sign reversal and difference in strength of $A_{\mathrm{SP}}$ between the $e^{-}$and $h$ parts of the YSR state are indeed theoretically expected and qualitatively reproduced [Fig. 4(c)] using the YSR simulation method [27]. Here, we assumed an impurity spin, which is coupled to the superconductor and described by the same magnetic anisotropy as given in the experimentally determined effective spin Hamiltonian above, but with a Zeeman term of $g \mu_{B} \hat{S}_{z} \cdot\left(B_{\text {tip }}+B_{\text {ext }}\right)$ in order to account for $B_{\text {tip }}$. The strengths of $J_{\text {eff }}$ and $U$ have been chosen such that a spectrum in one of the configurations of tip and $B_{\text {ext }}$ qualitatively fits the data [27]. Note that a quantitative comparison between the data and the simulation is hampered by the unknown magnetic anisotropy of the type $A$ atoms which can differ from that of the type $B$ atoms considered here for simplicity. However, the order of magnitude of the simulated asymmetry which assumes $\eta=0.1$ fits with the experimental data.

SPSTS in the four configurations of tip and $B_{\text {ext }}$, using a different microtip from the same macroscopic tip [27], has also been measured for a type $A$ atom with $A_{e-h}<0$ [Figs. 4(g,h)], and for one close to the QPT with only one peak close to $E_{F}$ [Figs. 4(d,e)]. They are compared to according simulations with $\left|J_{\text {eff }}\right| \ll\left|J_{\text {QPT }}\right|$ [Fig. 4(i)] and $\left|J_{\text {eff }}\right|=0.86\left|J_{\mathrm{QPT}}\right|$ [Fig. 4(f)], respectively. Consistently, the spin polarization at both bias polarities reverses its sign going from $A_{e-h}>0$ to $A_{e-h}<0$ in the experimental data, and from $\left|J_{\text {eff }}\right|>\left|J_{\mathrm{QPT}}\right|$ to $\left|J_{\text {eff }}\right|<\left|J_{\mathrm{QPT}}\right|$ in the simulations. Most interestingly, right at the QPT $\left(\left|J_{\text {eff }}\right|=\left|J_{\mathrm{QPT}}\right|\right)$ where the YSR state is exactly at $E_{F}$, the simulations 
predict a vanishing spin polarization [Fig. 4(k)]. These characteristic signatures of YSR states close to $E_{F}$ enable a clear distinction of the topologically trivial states from Majorana zero modes which can form at the end of chains made of YSR atoms [1,2], where a nonzero spin polarization with no sign change across $E_{F}$ is expected $[42,43]$.

In conclusion, we have experimentally verified the theoretically predicted spin polarization of the YSR state of a magnetic atom coupled to a superconductor across the QPT. Our results for the characteristic signatures of the spin polarization could help to unambiguously disentangle topologically nontrivial Majorana bound states from trivial YSR states using SPSTS.

We thank Markus Ternes for providing the code for the simulations of the spin excitation data and acknowledge fruitful discussions with Maria Valentyuk, Roberto Mozara, Alexander I. Lichtenstein, and Thore Posske. This work has been supported by the ERC Advanced Grant ASTONISH (No. 338802). L.C. and J.W. acknowledge support through the DFG priority program SPP1666 (Grant No. WI 3097/2). J. F. and S. P. acknowledge support from Vetenskapsrådet and Stiftelsen Olle Engkvist Byggmästare. A. A. K. would like to acknowledge financial support from the Emmy Noether Program (KH324/1-1) via the Deutsche Forschungsgemeinschaft.

*Corresponding author.

jwiebe@physnet.uni-hamburg.de

Corresponding author.

akamlapu@physnet.uni-hamburg.de

*Present address: Max Planck Institute for Solid State Research, D-70569 Stuttgart, Germany.

Corresponding author.

lzhou@physnet.uni-hamburg.de

${ }^{\S}$ Present address: Institute for Molecules and Materials (IMM), Radboud University, Nijmegen 6525 AJ, Netherlands.

[1] S. Nadj-Perge, I. K. Drozdov, J. Li, H. Chen, S. Jeon, J. Seo, A. H. MacDonald, B. A. Bernevig, and A. Yazdani, Science 346, 602 (2014).

[2] B. E. Feldman, M. T. Randeria, J. Li, S. Jeon, Y. Xie, Z. Wang, I. K. Drozdov, B. Andrei Bernevig, and A. Yazdani, Nat. Phys. 13, 286 (2017).

[3] M. Ruby, F. Pientka, Y. Peng, F. von Oppen, B. W. Heinrich, and K. J. Franke, Phys. Rev. Lett. 115, 197204 (2015).

[4] J. Li, T. Neupert, Z. Wang, A. H. MacDonald, A. Yazdani, and B. A. Bernevig, Nat. Commun. 7, 12297 (2016).

[5] G. C. Ménard et al., arXiv:1607.06353.

[6] L. Yu, Acta Phys. Sin. 21, 75 (1965).

[7] H. Shiba, Prog. Theor. Phys. 40, 435 (1968).

[8] A. I. Rusinov, Sov. Phys. JETP 29, 1101 (1969).

[9] J. D. Sau and P. M. R. Brydon, Phys. Rev. Lett. 115, 127003 (2015).

[10] A. Yazdani, B. A. Jones, C. P. Lutz, M. F. Crommie, and D. M. Eigler, Science 275, 1767 (1997).
[11] S.-H. Ji, T. Zhang, Y.-S. Fu, X. Chen, X.-C. Ma, J. Li, W.-H. Duan, J.-F. Jia, and Q.-K. Xue, Phys. Rev. Lett. 100, 226801 (2008).

[12] M. Ruby, Y. Peng, F. von Oppen, B. W. Heinrich, and K. J. Franke, Phys. Rev. Lett. 117, 186801 (2016).

[13] D.-J. Choi, C. Rubio-Verdú, J. de Bruijckere, M. M. Ugeda, N. Lorente, and J. I. Pascual, Nat. Commun. 8, 15175 (2017).

[14] N. Hatter, B. W. Heinrich, M. Ruby, J. I. Pascual, and K. J. Franke, Nat. Commun. 6, 8988 (2015).

[15] G. C. Ménard et al., Nat. Phys. 11, 1013 (2015).

[16] S. Kezilebieke, M. Dvorak, T. Ojanen, and P. Liljeroth, arXiv:1701.03288v1.

[17] K. J. Franke, G. Schulze, and J. I. Pascual, Science 332, 940 (2011).

[18] J. Bauer, J. I. Pascual, and K. J. Franke, Phys. Rev. B 87, 075125 (2013).

[19] M. Ruby, B. W. Heinrich, Y. Peng, F. von Oppen, and K. J. Franke, Nano Lett. 17, 4473 (2017).

[20] A. V. Balatsky, I. Vekhter, and J.-X. Zhu, Rev. Mod. Phys. 78, 373 (2006).

[21] M.E. Flatté and J. M. Byers, Phys. Rev. B 56, 11213 (1997).

[22] M.E. Flatté and J. M. Byers, Phys. Rev. Lett. 78, 3761 (1997).

[23] M. I. Salkola, A. V. Balatsky, and J. R. Schrieffer, Phys. Rev. B 55, 12648 (1997).

[24] P. Löptien, L. Zhou, A. A. Khajetoorians, J. Wiebe, and R. Wiesendanger, J. Phys. Condens. Matter 26, 425703 (2014).

[25] P. Berggren and J. Fransson, Europhys. Lett. 108, 67009 (2014).

[26] P. Berggren and J. Fransson, Phys. Rev. B 91, 205438 (2015).

[27] See Supplemental Material at http://link.aps.org/ supplemental/10.1103/PhysRevLett.119.197002 for details on the experimental and simulation techniques, the measurement of the substrate energy gap, and additional spectroscopic data and simulations, which includes Refs. [28-31].

[28] A. Schlenhoff, S. Krause, G. Herzog, and R. Wiesendanger, Appl. Phys. Lett. 97, 083104 (2010).

[29] Y. Smirnov and V. A. Finkel, Sov. J. Exp. Theor. Phys. 22, 750 (1966).

[30] J. Fransson, O. Eriksson, and A. V. Balatsky, Phys. Rev. B 81, 115454 (2010).

[31] S. Manna, A. Kamlapure, L. Cornils, T. Hänke, E. M. J. Hedegaard, M. Bremholm, B. B. Iversen, Ph. Hofmann, J. Wiebe, and R. Wiesendanger, Nat. Commun. 8, 14074 (2017).

[32] M. Ternes, W.-D. Schneider, J.-C. Cuevas, C. P. Lutz, C. F. Hirjibehedin, and A. J. Heinrich, Phys. Rev. B 74, 132501 (2006).

[33] M. Ruby, F. Pientka, Y. Peng, F. von Oppen, B.W. Heinrich, and K. J. Franke, Phys. Rev. Lett. 115, 087001 (2015).

[34] A. V. Titov and H. Jagodzinski, Surf. Sci. 152-153, 409 (1985).

[35] E. L. Wolf, D. M. Burnell, Z. G. Khim, and R. J. Noer, J. Low Temp. Phys. 44, 89 (1981). 
[36] L. Y. L. Shen, Phys. Rev. Lett. 24, 1104 (1970).

[37] B. W. Heinrich, L. Braun, J. I. Pascual, and K. J. Franke, Nat. Phys. 9, 765 (2013).

[38] A. A. Khajetoorians, T. Schlenk, B. Schweflinghaus, M. dos Santos Dias, M. Steinbrecher, M. Bouhassoune, S. Lounis, J. Wiebe, and R. Wiesendanger, Phys. Rev. Lett. 111, 157204 (2013).

[39] M. Ternes, New J. Phys. 17, 063016 (2015).
[40] S. Loth, C. P. Lutz, and A. J. Heinrich, New J. Phys. 12, 125021 (2010).

[41] F. Meier, L. Zhou, J. Wiebe, and R. Wiesendanger, Science 320, 82 (2008).

[42] P. Kotetes, D. Mendler, A. Heimes, and G. Schön, Physica (Amsterdam) 74E, 614 (2015).

[43] K. Björnson, S. S. Pershoguba, A. V. Balatsky, and A. M. Black-Schaffer, Phys. Rev. B 92, 214501 (2015). 